\title{
Simplified first-trimester fetal cardiac screening (four chamber view and ventricular oufflow tracts) in a low-risk population
}

\author{
Emanuela Orlandi ${ }^{1}$, Cinzia Rossi $^{2}$, Antonio Perino ${ }^{1}$, Giulia Musicò $^{2}$ and Francesco Orlandi ${ }^{2 *}$ \\ 'Department of Obstetric and Gynecology, University Hospital 'Paolo Giaccone', Palermo, Italy \\ ${ }^{2}$ Centre for Prenatal Diagnosis, Palermo, Italy \\ *Correspondence to: Francesco Orlandi. E-mail: orlandi@diagnosiprenatale.ił
}

\begin{abstract}
Objectives Our aim was to assess the accuracy of a simplified fetal cardiac study, inclusive of four-chamber view (4CV) and ventricular outflow tracts, performed during the 11-14 week screening by well-trained obstetricians to detect congenital heart diseases (CHDs).

Methods A transabdominal ultrasound was performed on 4820 singleton pregnant women at 11-14 weeks to visualize the visceral site, the $4 \mathrm{CV}$, and the outflow tracts. Neonatal outcomes were recorded 6 and 12 months after birth.

Results Among the 4820 patients reviewed, 790 were excluded because of loss at prenatal or postnatal follow-up (649 cases), or inability to obtain adequate first-trimester sonographic cardiac evaluation (141 cases). Among the 4030 included cases, 32 CHD cases were detected (20 major and 12 minor); 18 of the major $(90 \%)$ and five of the minor $(42 \%)$ were detected or suspected in the first trimester, one major and six minor in the second trimester, and one major and one minor only after birth.
\end{abstract}

Conclusions A simplified protocol is an effective tool to screen for CHD at 11-14 weeks. @ 2014 John Wiley \& Sons, Ltd.

Funding sources: None

Conflicts of interest: None declared

\section{INTRODUCTION}

Congenital heart diseases (CHDs) are the most common malformations, with a prevalence ranging between 5 and 9/1000 live births, and they are the leading cause of death due to malformation diseases in the first year of life. ${ }^{1-3}$ Ultrasonography is the gold standard for prenatal diagnosis of congenital malformations, ${ }^{4-6}$ and fetal echocardiography performed in the second and third trimester, also in high-risk populations, has a $60-100 \%$ sensitivity in diagnosing a major $\mathrm{CHD}^{7,8}$. The spread of first-trimester screening has allowed early diagnosis of various congenital malformations, ${ }^{9}$ including $\mathrm{CHD},{ }^{3,10-13}$ given that most cardiac malformations are already defined at this stage. The relationship between thick nuchal translucency (NT), altered ductus venosus (DV), and tricuspid regurgitation (TR $)^{14-17}$ with CHD has heightened the interest towards early fetal cardiac anomaly screening.

Early CHD detection has several advantages, namely reassuring high-risk women with normal ultrasound screening and performing early counseling in cases of pathological findings. Only few first-trimester fetal heart studies on low-risk populations have been published in literature, ${ }^{12,13,20-23}$ also because of the difficulty to perform a complete echocardiography in the first trimester. ${ }^{24}$ The aim of our study was to evaluate a new simplified approach to study the fetal hearth that can be used for all pregnancies undergoing first-trimester screening, visualizing only the visceral site, the four-chamber view $(4 \mathrm{CV})$, and the ventricular outflow tracts.

\section{METHODS}

This is a prospective study on an unselected population referred to the Centre for Prenatal Diagnosis of Palermo, Italy, between January 2009 and December 2011 for first-trimester screening of aneuploidies. The study included singleton pregnancies with fetal crown-rump length between 48 and $84 \mathrm{~mm}$. Genetic screening was based on maternal age, levels of maternal serum free beta human chorionic gonadotrophin (hCG), and pregnancy-associated plasma protein A (PAPP-A) on dried blood spot samples (DBS) (Life-Cycle, PerkinElmer), ${ }^{25}$ NT measurement, and presence of fetal nasal bone (NB), according to the Fetal Medicine Foundation (FMF) criteria. All women underwent weight and height measurements to calculate the body mass index (BMI) in $\mathrm{kg} / \mathrm{m} 2$. A transabdominal (TA) ultrasound screening was performed by three experienced sonographers holding an FMF certificate of competence for measurement of NT, NB, TR, and DV, using Voluson E8 (GE Medical Systems, Zipf, Austria) ultrasound machines with RAB 4-8D probes. Customized settings were used 
for first-trimester fetal heart scan, minimizing ultrasound exposure of the fetus, especially in the Doppler mode. In 202 patients, with substandard quality TA images, we performed an additional transvaginal (TV) ultrasound examination using an IC59HD probe.

During the test, in addition to measuring the NT, assessing NB presence/absence, and observing several other fetal anatomical structures according to the FMF protocol, instead of a complete echocardiography, we followed a simplified protocol to acquire specific information on the cardiac morphology attempting to visualize the following:

- the visceral site and the position of the heart;

- the four chambers, their size, and the atrio-ventricular (AV) valves;

- the origin of the aorta from the left ventricle, the origin of the pulmonary artery from the right ventricle, and the vessels crossing.

To increase accuracy and reduce the duration of the test, we used color flow mapping to visualize the transvalvular flow and its malfunctions, if any, and the appropriate filling of both ventricles (Figure 1), as well as the flow in the aortic and pulmonary artery (Figures 2 and 3 ).

Cases reviewed in the first trimester were classified as apparently normal (3988), pathological (20), or suspicious (22). The last group included cases where imaging, albeit suspicious, failed to allow a precise definition or a clear-cut distinction between physiology and pathology (e.g. apparent disproportion between ventricles). In cases deemed normal, the fetal heart scan required very limited additional time (never more than $10 \mathrm{~min}$ ).

A follow-up scan was performed at 20-22 weeks to assess the cardiac structure. In abnormal cases, we recommended fetal karyotyping by villocentesis and scheduled a follow-up 2-3 weeks later. In cases considered suspicious, the cardiac evaluation was repeated at 15 weeks of gestation.

Fetal cardiac defects were defined as major, if they were either lethal or required surgery or interventional cardiac catheterization during the first year of life in the newborn, and minor, if they were generally asymptomatic. For the

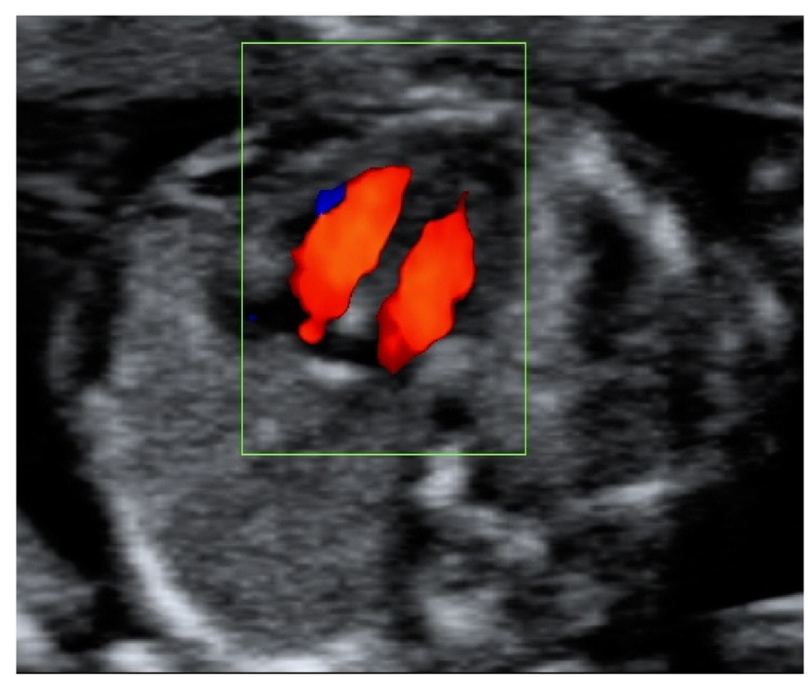

Figure 1 Four-chamber view, transvalvular flow at 12 weeks

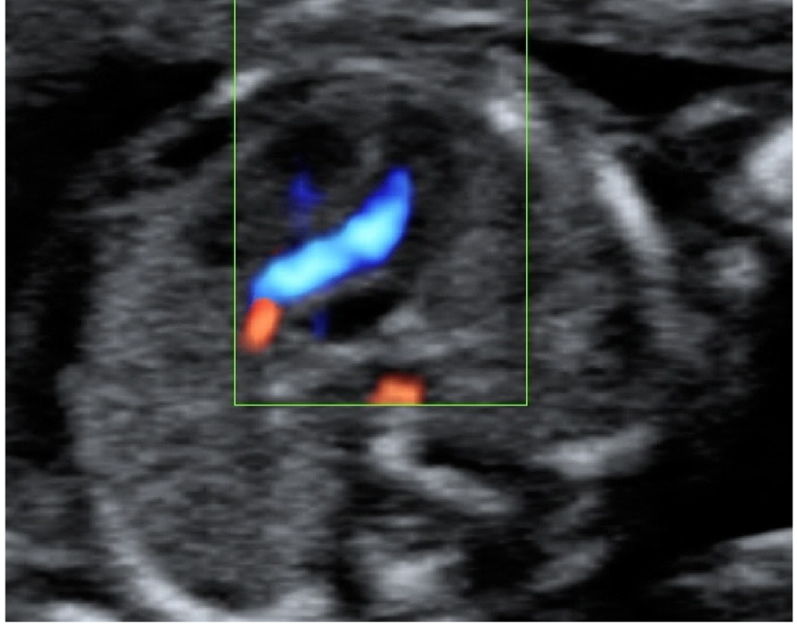

Figure 2 Left outflow tract at 12 weeks

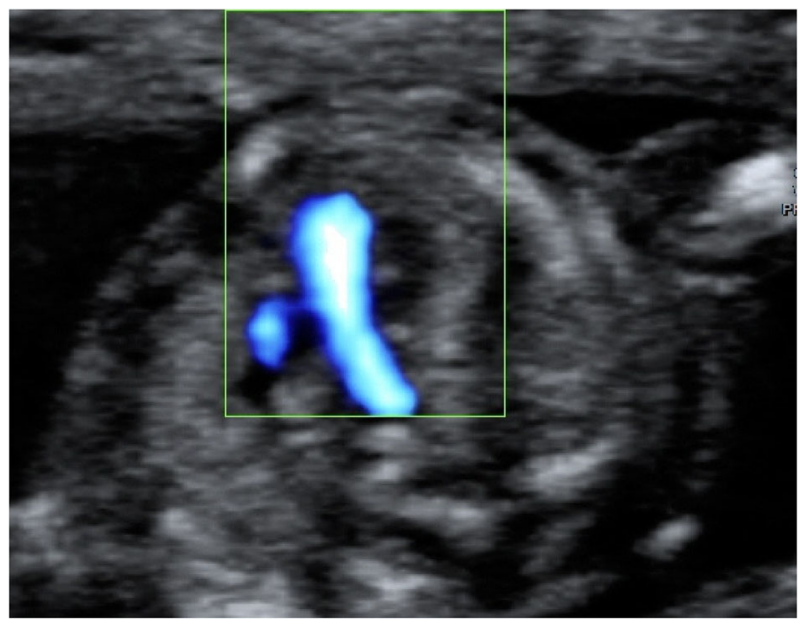

Figure 3 Pulmonary trunk flow at 12 weeks

purpose of this study, TR was not considered a CHD as it is normally functional and transitory.

If the quality of the images was unsatisfactory, the patient underwent a TV ultrasound examination. The clips and volumes collected in each case considered pathological or suspicious were subsequently reviewed by a pediatric cardiologist.

The parents were informed of the potential limitation of this ultrasound screening, a technique still under evaluation, and of the possibility of obtaining an uncertain picture that could cause maternal anxiety while waiting for a more precise diagnosis.

Follow-up at 6 and 12 months after birth was obtained on the basis of the information provided with the clinical examination by a neonatologist at birth, by the mothers, or their gynecologists. We chose to seek information also from the gynecologist because in Sicily, most of women are treated during the pregnancy, delivery, and post-partum by the same gynecologist, which has therefore reliable information on the child's health. In cases where the infant underwent surgery, pertinent information was provided by the pediatric surgeon. 


\section{RESULTS}

Of the patients examined during the study period $(4820$ singleton pregnancies), 649 were excluded because of lost to second-trimester follow-up or at delivery. Additional 141 cases were excluded because of failure to appropriately visualize the cardiac morphology both with a TA and TV approach $(41 / 686$ or $6 \%$ cases at 11 weeks, $58 / 1871$ or $3.1 \%$ at 12 weeks, and $42 / 1614$ or $2.6 \%$ at 13 weeks). The remaining population included 4030 pregnancies, 3969 studied with a TA scan and 61 also with a TV scan. The average maternal age was 33 years (range 16-43 year), weight was $65.7 \mathrm{~kg}$ (range $41-121 \mathrm{~kg}$ ), gestational age was 12.5 weeks, and BMI $24.5 \mathrm{~kg} / \mathrm{m}^{2}$ (range $17.5-43.3 \mathrm{~kg} / \mathrm{m}^{2}$ ). Gestational age at the examination was 11 + weeks in 645 pregnancies (16\%), 12+ weeks in 1813 (45\%), and 13+weeks in 1572 (39\%). Screening was considered appropriate when it provided a satisfactory view of the four chambers, the AV valves, and the origin and the crossing of the large arteries. Color Doppler was used in a complementary manner to visualize the chambers, the great vessels, and the direction of flows.

Following an insufficient TA scan (202 cases), an additional TV examination provided information on 61 women: at 11 weeks in $27(4 \%)$, at 12 weeks in $21(1.2 \%)$, and at 13 weeks in 13 cases $(0.8 \%)$. The main factors hampering an appropriate visualization of the cardiac structures were a high BMI and a considerably early gestational age, as reported also by previous studies. ${ }^{10-12}$ BMI was significantly greater for women in whom both TA and TV examinations were unsatisfactory compared with those in whom it was successful (30.2 vs $\left.24.5 \mathrm{~kg} / \mathrm{m}^{2}, \quad p<0.001\right)$. The mean crown-rump length was $65 \mathrm{~mm}$ in successful cases versus $58 \mathrm{~mm}$ in unsuccessful cases $(p<0.001)$.

Among the 4030 fetuses included in the study there were 32 CHD cases (0.8\%), 20 major and 12 minor (Table 1); 16 of the major and five of the minor cases were detected in the first trimester.

The most common major abnormality diagnosed was atrioventricular septal defect (AVSD) (Figure 4), seven cases, and hypoplastic left heart syndrome (HLHS) (Figure 5), four cases.

Women carrying a fetus with major CHD were offered villocentesis or, at a later stage, amniocentesis in cases ascertained in the second trimester, to determine the fetal karyotype, but four of them opted for voluntary termination of pregnancy (TOP) without undergoing additional examinations (case 15 also showed fetal hydrops and case 21 an occipital encephalocele). Of the 15 fetuses with major CHD undergoing fetal karyotyping, nine $(60 \%)$ presented an aneuploidy and the pregnancy was terminated; two of them, cases 30 and 31 in Table 1, presented a complex CHD difficult to interpret, even by a pediatric cardiologist. In the second trimester, a CHD was confirmed in all the remaining six fetuses although, in one case, the pediatric cardiologist changed the diagnosis made in the first trimester (case 28) (Figure 6) from single ventricle + truncus arteriosus to single ventricle + double outlet right ventricle (DORV). Of the other four major CHDs, one deemed normal in the first trimester proved to be Tetralogy of Fallot (TOF) at 20 weeks; two classified as suspicious were diagnosed as pathological at 15 weeks (case 23 HLHS and case
32 DORV), whereas the last major CHD (aortic coarctation) was detected only after birth. Six of the seven minor CHD not detected in the first trimester, five ventricular septal defect (VSD) and one moderated chamber disproportion, were identified in the second trimester, and one VSD was detected only after birth. Twenty of the 22 suspicious cases proved to be normal at 15 and 20 weeks. There were no false positives among the major CHD cases.

The clips and volumes collected for each fetus considered pathological or suspicious were subsequently reviewed by a pediatric cardiologist who agreed with all diagnoses except that in case 28.

Of the 4030 fetuses included in our study, 205 (5.1\%) had a NT $>95$ th percentile. The NT was higher than the 95th percentile for gestational age in six of the 20 major CHD (30\%) and higher than the 99th percentile in five additional cases, suggesting that an elevated NT can be an effective marker of major CHD. Only in one of the 11 minor CHDs was the NT abnormal (higher than the 99th percentile).

Aneuploidy screening results were positive in all seven cases of Trisomy 21 and in four of the five Trisomy 18, and there were no cases of Trisomyl3.

No additional relevant CHDs were detected at follow-up 6 and 12 months after birth.

\section{DISCUSSION}

Fetal echocardiography is usually performed in the second trimester of pregnancy in patients with a high risk of carrying a fetus affected by a CHD although the majority of infants with a CHD have no previous risk factors. ${ }^{26}$ This study demonstrates the possibility to use a simplified protocol to perform a CHD screening during the first-trimester TA ultrasound scan for NT measurement.

There are several good reasons to attempt early CHD detection: differently from other organs, for example, the brain, at this stage, the fetal heart has almost fully developed; compared with other malformations, CHD have a higher prevalence $^{27,28}$ and can be associated with chromosome anomalies.

Over the years, a correlation has been documented, albeit with modest sensitivity in euploid fetuses, ${ }^{14,19,30}$ between high NT and CHD. ${ }^{30}$ In the same way, other first-trimester markers of aneuploidy such as TR and DV ${ }^{13-18}$ are associated with CHD. This explains the growing interest in early study of cardiac morphology, enhanced by the progress of ultrasound technology and by the growing skill of operators performing first-trimester screening. In recent years, this has allowed to assess the cardiac morphology in patients at low risk ${ }^{12,13,20}$ and high risk ${ }^{3-11}$ using a TA approach.

These tests require high-level ultrasound equipment, dedicated settings, especially for color flow mapping, essential for correct visualization in cases of low echogenicity, and considerable experience of operators studying fetal morphology.

Our data demonstrate how appropriately trained operators can correctly identify most of the major CHD already in the first trimester. Our 11-14 week simplified fetal cardiac screening allowed us to detect (16) or suspect (2) 18 out of 
Table 1 Cases of congenital heart disease

\begin{tabular}{|c|c|c|c|c|c|c|c|c|}
\hline$\#$ & Cardiac defect & Weeks & First trimester & Second trimester & Outcome & K & NT & Screening risk \\
\hline \multicolumn{9}{|c|}{ Minor defects } \\
\hline 1 & VSD & 12 & VSD & VSD & OK & $\mathrm{NL}$ & 2.4 & $\mathrm{HIGH}$ \\
\hline 2 & VSD & 13 & VSD & VSD & OK & $\mathrm{NL}$ & 2.2 & LOW \\
\hline 3 & VSD & 13 & NL & VSD & OK & $\mathrm{NL}$ & 2.0 & LOW \\
\hline 4 & VSD & 12 & NL & VSD & OK & $\mathrm{NL}$ & 2.4 & LOW \\
\hline 5 & VSD & 12 & NL & $\mathrm{NL}$ & OK & $\mathrm{NL}$ & 1.5 & LOW \\
\hline 6 & VSD & 12 & NL & VSD & OK & $\mathrm{NL}$ & 1.6 & LOW \\
\hline 7 & VSD & 13 & NL & VSD & OK & $\mathrm{NL}$ & 1.9 & LOW \\
\hline 8 & VSD & 13 & NL & VSD & OK & $\mathrm{NL}$ & 2.0 & LOW \\
\hline 9 & $\mathrm{LV}<\mathrm{RV}$ & 11 & $\mathrm{LV}<\mathrm{RV}$ & $\mathrm{LV}<\mathrm{RV}$ & IUD 20 weeks & $\mathrm{NL}$ & $10^{a}$ & $\mathrm{HIGH}$ \\
\hline 10 & $\mathrm{LV}<\mathrm{RV}$ & 12 & NL & $\mathrm{LV}<\mathrm{RV}$ & OK & $\mathrm{NL}$ & 1.6 & LOW \\
\hline 11 & $R A>L A$ & 13 & $R A>L A$ & $R A>L A$ & OK & $\mathrm{NL}$ & 1.9 & LOW \\
\hline 12 & Rt Ao Arch & 13 & Rt Ao Arch & Rt Ao Arch & OK & $\mathrm{NL}$ & 2.2 & LOW \\
\hline \multicolumn{9}{|c|}{ Major defects } \\
\hline 13 & AVSD & 12 & AVSD & & TOP & $\mathrm{T} 18$ & $4.5^{\circ}$ & $\mathrm{HIGH}$ \\
\hline 14 & AVSD & 12 & AVSD & & TOP & T21 & $8.0^{a}$ & $\mathrm{HIGH}$ \\
\hline 15 & AVSD & 12 & AVSD & & TOP & & $9.3^{a}$ & $\mathrm{HIGH}$ \\
\hline 16 & AVSD & 12 & AVSD & & TOP & T21 & $3.3^{b}$ & $\mathrm{HIGH}$ \\
\hline 17 & AVSD & 12 & AVSD & & TOP & T21 & $5^{a}$ & $\mathrm{HIGH}$ \\
\hline 18 & AVSD & 12 & AVSD & AVSD & AAS & OK & $2.9^{b}$ & LOW \\
\hline 19 & AVSD & 12 & AVSD & & TOP & $\mathrm{T} 18$ & $3.1^{b}$ & $\mathrm{HIGH}$ \\
\hline 20 & TGA & 13 & TGA & TGA & AAS & OK & 2.0 & LOW \\
\hline 21 & HLHS & 12 & HLHS & & TOP & & 1.2 & LOW \\
\hline 22 & HLHS & 13 & HLHS & & TOP & & 1.8 & LOW \\
\hline 23 & HLHS & 13 & SUSP. & HLHS & DAS & OK & 2.5 & LOW \\
\hline 24 & HLHS & 13 & HLHS & & TOP & & $3.2^{b}$ & LOW \\
\hline 25 & TOF & 12 & NL & TOF & AAS & OK & 1.5 & LOW \\
\hline 26 & PA stenosis & 13 & PA stenosis & PA stenosis & DAS & OK & 2.5 & $\mathrm{HIGH}$ \\
\hline 27 & TR atresia & 13 & TR atresia & TR atresia & TOP & T21 & $2.9^{b}$ & $\mathrm{HIGH}$ \\
\hline 28 & Single ventricle + DORV & 12 & Single Ventr. + Truncus & Single Ventr. + DORV & TOP & $\mathrm{T} 18$ & $3.1^{b}$ & LOW \\
\hline 29 & Ao Coartation & 13 & NL & $\mathrm{NL}$ & AAS & & 2.2 & LOW \\
\hline 30 & Complex CHD & 12 & ¿ุ? & & TOP & $\mathrm{T} 18$ & $3.7^{a}$ & $\mathrm{HIGH}$ \\
\hline 31 & Complex CHD & 13 & ใ? & & TOP & $\mathrm{T} 13$ & 2.5 & LOW \\
\hline 32 & DORV & 13 & SUSP. & DORV & AAS & OK & 2.4 & LOW \\
\hline
\end{tabular}

K, karyotype; AAS, alive after surgery; Ao coartation, coartation of the aorta; AVSD, atrioventricular septal defect; IUD, intra-uterine death; LA, left atrium; LOW, low risk; LV, left ventricle; NL, normal; NT, nuchal translucency; RA, right atrium; Rt Ao Arch, right aortic arch; RV, right ventricle; SUSP., suspicious; TGA, transposition of the great arteries; TOF, Tetralogy of Fallot; TOP, termination of pregnancy; TRUNCUS., truncus arteriosus; VSD, ventricular septal defect.

${ }^{a} \mathrm{NT}$ at $>99+$ th percentile.

${ }^{b} N T$ at $>95$ th percentile.

20 major CHD in the first trimester, with a $90 \%$ sensibility and a $99.5 \%$ specificity. The most frequent major CHD in our series, as shown in Table 1, is represented by AVSD, seven cases, and among these, three fetuses were affected by Trisomy 21 and two by Trisomy 18, confirming the strong association between these anomalies and aneuploidies. In four cases, we detected HLHS, but in three of these, the parents decided to terminate the pregnancy declining an invasive procedure. Among the minor CHDs, there were seven VSDs, which are confirmed as the most frequent congenital heart malformation. ${ }^{1}$

The 4CV alone, relatively easy to obtain and representing the basis to assess, for example, the presence of TR, allowed us, also using the color Doppler, to detect 15 of the 20 major CHD (75\%). We believe that direct observation of the cardiac morphology is more effective, 


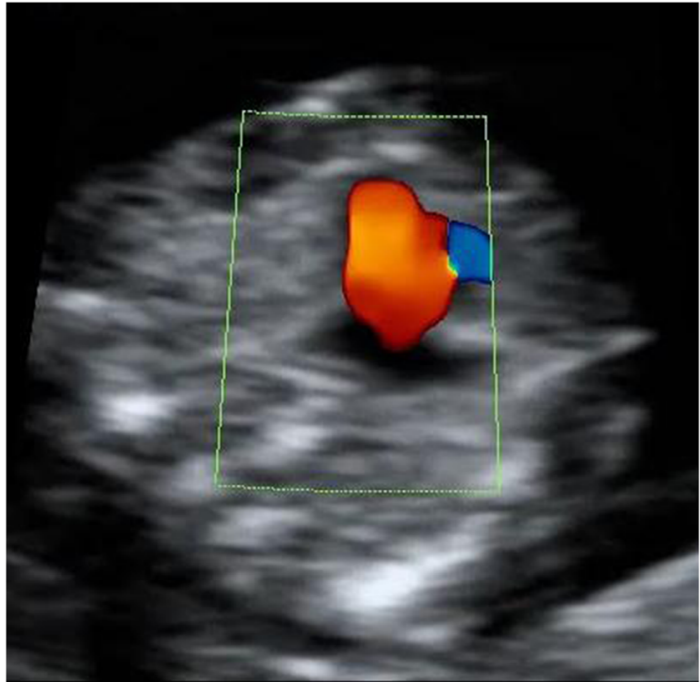

Figure 4 Color Doppler image demonstrating a complete atrioventricular septal defect

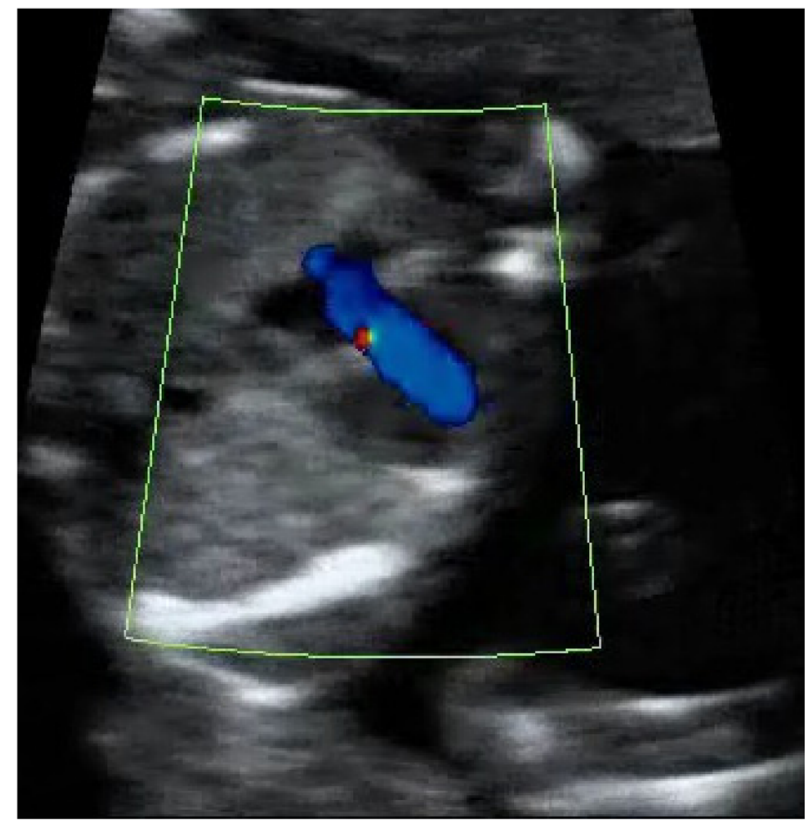

Figure 5 Color Doppler image demonstrating single flow through the tricuspid valve (hypoplastic left heart syndrome)

at least for the structures more easily visualized using our suggested simplified protocol, than searching for indirect CHD markers such as DV and TR. In addition, we believe that a CHD screening following this simplified protocol is by no means technically more complex than studying the VD or TR currently used as risk markers for CHD and aneuploidies.

The major CHDs are already present, and detectable in most cases, in the first trimester although between the first and second trimesters, some cases present different evolutionary features. For example, complete AV canal defects can be

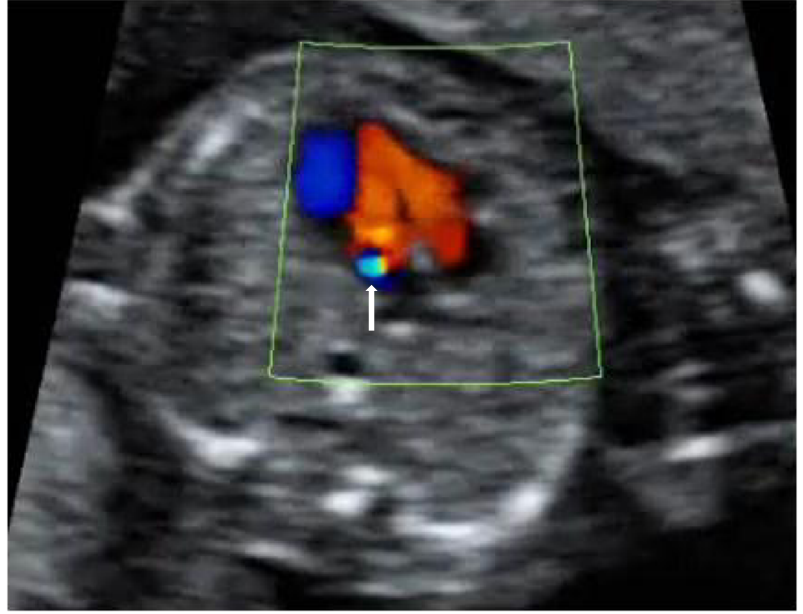

Figure 6 Single ventricle + double outlet right ventricle (case 28): color Doppler image demonstrating the single ventricle and mitral regurgitation (arrow)

easily detected if appropriately investigated, others such as the Tetralogy of Fallot are more insidious, and others such as aortic coartation are difficult to diagnose at an early stage because of their history and natural evolution. Other scenarios, such as chamber disproportions, may be more difficult to interpret - they can evolve overtime for better or for worse - and call for additional investigation in terms of reference values and clinical significance. On the other hand, second-trimester fetal morphology screening still stands as essential.

As in several published works, ${ }^{3-13,19}$ a limitation of this study is the unavailability of post-mortem examination in cases in which the patients decided for TOP. The echocardiography and postnatal surgery, when required, did however confirm the diagnosis in the other cases.

A major CHD is frequently associated to chromosomal anomalies, as reported by other studies, ${ }^{2-10,12}$ with a very low incidence of false positives. In our study, nine of 16 fetuses affected by a major CHD (60\%) were aneuploidic, and in the absence of fetal karyotyping, we cannot rule out other cases of chromosomal anomalies in the four cases of TOP. On the other hand, out of the 13 fetuses with a chromosomal anomaly included in our sample, nine $(69 \%)$ had a major CHD. Given that the fetuses with a major CHD and a normal karyotype in our group were only seven (we are unaware of the karyotype of four fetuses with major $\mathrm{CHD}$ as the pregnancies were terminated), detecting a major $\mathrm{CHD}$ in the first trimester proves to be an effective marker of chromosomal disorders with a $69 \%$ sensibility and a $99.8 \%$ specificity.

\section{CONCLUSION}

An obstetrician with good experience in second-trimester fetal heart screening can distinguish most, but not all, of the normal findings from the abnormal ones, visualizing the $4 \mathrm{CV}$ and ventricular outflows in the first trimester of pregnancy. 
An important goal of the first-trimester cardiac scan is to differentiate normal cases from those that require subsequent investigation and the assistance of a pediatric cardiologist.

Early CHD detection in the first trimester will allow to reassure mothers with healthy fetuses, to perform a short-tomedium-term follow-up in suspicious cases, to provide appropriate counseling in the presence of cardiac anomalies, allowing the option of TOP at an earlier stage and the possibility to detect associated chromosomal disorders.

A fetal cardiac scan using a simplified protocol such as the one we describe can become an essential part of first-trimester ultrasound screening of all fetuses.

\section{REFERENCES}

1. van der Linde D, Konings E, Maarten A, et al Birth prevalence of congenital heart disease worldwide. JACC 2011;58-21:2241-7

2. Chaoui R, Heling KS, et al. Aberant right succlavian artery as a new cardiac sign in second and third trimester fetuses with Down syndrome. Am J Obstet Gynecol 2005;192:257-63.

3. Persico N, Moratalla J, Lombardi CM, et al. Fetal echocardiography at 11-13 weeks by transabdominal high-frequency ultrasound. Ultrasound Obstet Gynecol 2011;37:296-301

4. Kleinman CS, Hobbins JC, Jaffe CC, et al. Echocardiographic studies of the human fetus: prenatal diagnosis of congenital heart disease and cardiac dysrhythmias. Pediatrics 1980;65:1059-67

5. DeVore GR, Donnerstein RL, Kleinman CS, et al. Fetal echocardiography. I. Normal anatomy as determined by real-timedirected M-mode ultrasound. Am J Obstet Gynecol 1982;144:249-60.

6. DeVore GR. The prenatal diagnosis of congenital heart disease - a practical approach for the fetal sonographer. J Clin Ultrasound 1985;13:229-45.

7. Buskens E, Grobbe DE, Frohn MI, et al. Efficacy of routine fetal ultrasound screening for congenital heart disease in normal pregnancy. Circulation 1996;94:67-72

8. Hsieh CC, Kuo DM, Chiu TH, et al. Prenatal diagnosis of major congenital cardiovascular malformation. Gynecol Obstet Invest 1996;354:1242-7

9. Souka AP, Pilalis A, Kavalakis Y, et al. Assessment of fetal anatomy at the 11-14 week ultrasound examination. Ultrasound Obstet Gynecol 2002;24(7):730-4

10. Carvalho JS. 2004. Fetal heart scanning in the first trimester. Prenat Diagn 24(13):1060-7

11. Huggon IC, Ghi T, Cook AC, et al. Fetal cardiac abnormalities identified prior to 14 weeks' gestation. Ultrasound Obstet Gynecol 2002;20(1):22-9

12. Lombardi CM, Bellotti M, Fesslova V, et al. Fetal echocardiography at the time of the nuchal translucency scan. Ultrasound Obstet Gynecol 2007;29:249-57

13. Volpe P, Passamonti U, Volpe N, et al. Fetal cardiac evaluation at 11-14 weeks by experienced obstetricians in a low risk population. Prenat Diagn 2011;31:1054-61

14. Makrydimas G, Sotoriadis A, Huggon IC, et al. Nuchal translucency and fetal cardiac defects: a pooled analysis of major fetal echocardiography centers. Am J Obstet Gynecol 2005;192:89-95

15. Maiz N, Plasencia W, Dagklis T, et al. Ductus venosus Doppler in fetuses with cardiac defects and increased nuchal translucency thickness. Ultrasound Obstet Gynecol 2008;31:256-60

16. Huggon IC, DeFigueiredo DB, Allan LD. Tricuspid regurgitation in the diagnosis of chromosomal anomalies in the fetus at 11-14 weeks of gestation. Heart 2003;89:1071-3

17. Falcon O, Faiola S, Huggon I, et al. Fetal tricuspid regurgitation at the $11+0$ to $13+6$ week scan: association with chromosomal defects

\section{WHAT'S ALREADY KNOWN ABOUT THIS TOPIC?}

- First-trimester screening allowes early diagnosis of various congenital malformations, including congenital heart disease.

- Few first-trimester fetal heart studies on low-risk populations are published because of the difficulty to perform a complete echocardiography in the first trimester.

WHAT DOES THIS STUDY ADD?

- This study demonstrates how possible it is to detect most cardiac abnormalities by using a simplified protocol to screen for congenital heart disease during the first-trimester scan. and reproducibility of the method. Ultrasound Obstet Gynecol 2006;27:609-12, 16.

18. Timmermann E, Clur SA, Pajkrt E, et al. First-trimester measurement of the ductus venosus pulsatility index and prediction of congenital heart defects. Ultrasound Obstet Gynecol 2010;36:668-75

19. Bellotti M, Fesslova V, De Gasperi C, et al. Reliability of the first trimester cardiac scan by ultrasound-trained obstetricians with highfrequency transabdominal probes in fetuses with increased nuchal translucency. Ultrasound Obstet Gynecol 2010;36:272-8.

20. Rustico MA, Benettoni A, D'Ottavio G, et al. Early screening for fetal cardiac anomalies in an unselected population: the role of operator experience. Ultrasound Obstet Gynecol 2000;16:614-9

21. Schwarzler P, Carvalho JS, Senat MV, et al. Screening for fetal aneuploidies and fetal cardiac abnormalities by nuchal translucency thickness measurement at 10-14 weeks of gestation as part of routine antenatal care in an unselected population. Br J Obstet Gynaecol 1999;106:1029-34

22. Bronshtein M, Zimmer EZ. The sonographic approach to the detection of fetal cardiac anomalies in early pregnancy. Ultrasound Obstet Gynecol 2002;19:360-5

23. Vimpelli T, Huhtala H, Acharya G. Fetal echocardiography during routine first-trimester screening: a feasibility study in an unselected population. Prenat Diagn 2006;26:475-82

24. Carvalho JS. Screening for heart defects in the first trimester of pregnancy: food for thought. Ultrasound Obstet Gynecol 2010;36:658-660 yres NA. Advances in fetal echocardiography. Tex Heart Inst J 1997;24(4):250-9

25. Krantz D, Hallahan T, Ravens R. First trimester Down syndrome screening with dried blood spots using a dual anlayte free beta hCG and PAPP-A immunofluorimetric assay. Prenat Diagn 2011; Jun 21. doi: 10.1002/pd.2792.

26. Garne E, Stoll C, Clementi M. Evaluation of prenatal diagnosis of congenital heart diseas by ultrasound: experience from 20 European registries. Ultrasound Obstet Gynecol 2001;17:386-91

27. Garne E, Loane M, Dolk H, et al. Prenatal diagnosis of severe structural congenital malformation in Europe. Ultrasound Obstet Gynecol 2005;25:6-11

28. Hyett J. Does nuchal translucency have a role in fetal cardiac screening? Prenat Diagn 2004;24:1130-5

29. Atzei A, Gajewska K, Huggon IC, et al. Relation between nuchal translucency thickness and prevalence of major cardiac defcts in fetuses with normal karyotype. Ultrasound Obstet Gynecol 2005;26:154-7

30. Hyett J, Perdu M, Sharland G, et al. Using fetal nuchal translucency to screen for major congenital cardiac defects at 10-14 weeks of gestation: population based cohort study. BMJ 1999;318:81-5 